\title{
Modern treatment of perineuriomas: a case-series and systematic review
}

\author{
Anne-Kathrin Uerschels ${ }^{1}$, Christos Krogias ${ }^{2}$, Andreas Junker ${ }^{3}$, Ulrich Sure ${ }^{1}$, Karsten H. Wrede ${ }^{1}$ and \\ Oliver Gembruch" ${ }^{*}$ (D)
}

\begin{abstract}
Background: Perineuriomas are rare benign peripheral nerve sheath tumours of perineurial cell origin and can be classified into intraneural and extraneural perineuriomas. They most commonly present a mononeuropathy of gradual onset and slow progression, resulting in progressive neurological deficits like hypoesthesia or motor weakness. Therapy is still variable. Aim of the study was to compare our surgical treatment and our follow-up regime including high-resolution nerve sonography with the current literature to evaluate best treatment of perineuriomas.
\end{abstract}

Methods: Retrospective analysis of our dataset "peripheral nerve lesion" to identify patients suffering from perineuriomas between 01.01.2012 until 31.12.2018. Surgical treatment and the follow-up examination of three patients were described. Additionally, a systematic review including PubMed, the Cochrane Collaboration Library, Scopus and Google Scholar was performed for literature published between January 1, 1990 and October 31, 2019 independently by 2 authors.

Results: In the first case, the left ulnar nerve was affected. In the second case, the left peroneal nerve and in the third case the right median nerve was affected. High-resolution nerve sonography was performed in each case. All patients underwent interfascicular neurolysis combined with a targeted fascicular biopsy under electrophysiological monitoring. Neurological deficits improved subsidized by rehabilitation. Surgical therapy and the neurological outcome were compared with literature. Systematic review revealed 22 articles, which met the inclusion criteria. Therefore, demographics, surgical treatment and neurological outcome of 77 patients were analysed.

Conclusions: Perineuriomas are rare benign nerve sheath tumours with a slow progression, sometimes difficult to diagnose. Decompression and neurolysis may improve neurological deficits. High resolution nerve sonography might serve as a helpful additional diagnostic tool in this process.

Keywords: Intraneural perineurioma, Surgical treatment, Target fascicular biopsy, Decompression and neurolysis

\section{Background}

Perineuriomas $(\mathrm{PN})$ are rare benign peripheral nerve sheath tumours originating from perineurial cells. Intraneural and extraneural PN have to be distinguished. The intraneural PN is restricted to the boundaries of the peripheral nerve, whereas the extraneural $\mathrm{PN}$ is found mainly in soft tissues and skin [1].

They arise from perineural cells located in the perineurium of the peripheral nerve, which is

\footnotetext{
* Correspondence: oliver.gembruch@uk-essen.de

'Department of Neurosurgery, University Hospital Essen, University of

Duisburg-Essen, Essen, Germany

Full list of author information is available at the end of the article
}

histologically characterized by a complex perineurial cell proliferation, which extends into the endoneurium and surrounds concentrically individual myelinated and the unmyelinated axon-Schwann cell complexes of peripheral nerve fascicles and endoneurial capillaries. This leads to the characteristic "pseudo-onion bulbs" [2]. It is known that the perineurium forms the blood-nerve barrier and has continuity with the pia-arachnoid membrane of the central nervous system $[3,4]$. A neoplastic origin for intraneural and soft tissue $\mathrm{PN}$ is suggested due to the presence of $22 \mathrm{q}$ deletions $[5,6]$. However, recently performed genomic analysis indicates 
divergent pathogenetic mechanisms of intraneural $\mathrm{PN}$ and soft tissue PN. The intraneural PN frequently contains TRAF7 mutations [7], and rarely, chr22q12 deletions whereas the soft tissue PN showed no TRAF7 mutations. Alterations in NF1 or NF2 likely contribute to perineurioma pathogenesis, similar to other nerve sheath tumours [8].

They most commonly present a mononeuropathy of gradual onset and slow progression, resulting in progressive neurological deficits like hypoesthesia or motor weakness $[9,10]$. It is believed that the motoric fascicles are affected by the tumour, there as the sensory deficits are caused by the intraneural compression of the sensoric fascicles.

Those lesions can be difficult to diagnose due to their rarity. Up to now, guidelines do not exist, and the management of this rare pathology is still various.

Therefore, we retrospectively analysed our dataset "peripheral nerve lesion" to identify patients suffering from perineuriomas. Aim of the study was to compare our surgical treatment and our follow-up regime including high-resolution nerve sonography (HRNS) with the current literature.

\section{Methods}

\section{Patients}

Retrospective analysis of our dataset "peripheral nerve lesion" was performed between 01.01.2012 until 31.12.2018. Three patients suffering from an intraneural PN were identified. Patients were treated in our department using following surgical technique: microsurgical interfascicular neurolysis in combination with a biopsy of a non-functional fascicle. Medical records, pre- and postoperative HRNS and MRI were analysed. Follow-up examination and postoperative HRNS were evaluated up to $2 \frac{1}{2}$ years after surgery.

The study was approved by the Institutional Review Board (Medical Faculty, University of Duisburg-Essen, Registration number: 18-7955-BO).

\section{Surgery}

Preoperative HRNS was used to identify the affected nerve and to plan the extend of the skin incision followed by sterile washing and draping of the surgical field. After skin incision, careful preparation towards the nerve was performed. The affected nerve can be identified by its pathologically changed surface structure with an enlarged epineurium that covers the thickened fascicles. Intraoperative HRNS was now used to visualise the affected nerve and to plan the extend of the epineural opening. Microsurgical interfascicular neurolysis can be achieved after careful longitudinal incision of the epineurium. Electrophysiological stimulation (0.1$0.2 \mathrm{~mA}$ ) of each fascicle shows the ones with motor function. An enlarged fascicle without motor function is identified for a targeted fascicular biopsy (4 $\mathrm{mm}$ length). If possible, end-to-end anastomosis should be performed afterwards to prevent microneuroma.

\section{Systematic review of the literature}

A systematic search via PubMed, the Cochrane Collaboration Library, Scopus and Google Scholar was performed for literature published between January 1, 1990 and October 31, 2019 independently by 2 authors.

Search key words comprised "intraneural perineurioma", "intraneural perineuriomas" and "localized hypertrophic mononeuropathy". Inclusion criteria were articles published in English presenting the clinical course, peripheral nerve location, and the treatment regime. Therefore, cases without description of the surgical treatment were excluded.

Afterwards, the reference lists of included articles were reviewed to identify and include additional eligible articles. Furthermore, all included studies were meticulously cross-referenced to ensure that patients were not included in multiple articles (Fig. 1). The systematic review was conducted following Preferred Reporting Items for Systematic Reviews and MetaAnalyses guidelines [11].

\section{Statistical analysis}

Data were analysed using SPSS 23.0 (Statistical Package for the Social Sciences, SPSS Inc., Chicago, IL, USA). Metric data were described by mean and standard deviation and nominal data by frequency and valid percent.

\section{Results \\ Case presentations \\ Case 1}

A 56-year-old male patient with typical clinical and electroneurographic findings of carpal-tunnelsyndrome caused by an intraneural $\mathrm{PN}$ of the right median nerve presented to our neurosurgical department. The patient complaint about a progressive hypaesthesia and paresis of the hand and underwent 4 surgeries over a period of 30 years without long term benefit because of suspected carpal-tunnelsyndrome. HRNS and magnetic resonance imaging (MRI) of the right-hand depicted an intraneural PN of the median nerve (Fig. 2). Follow-up examination 


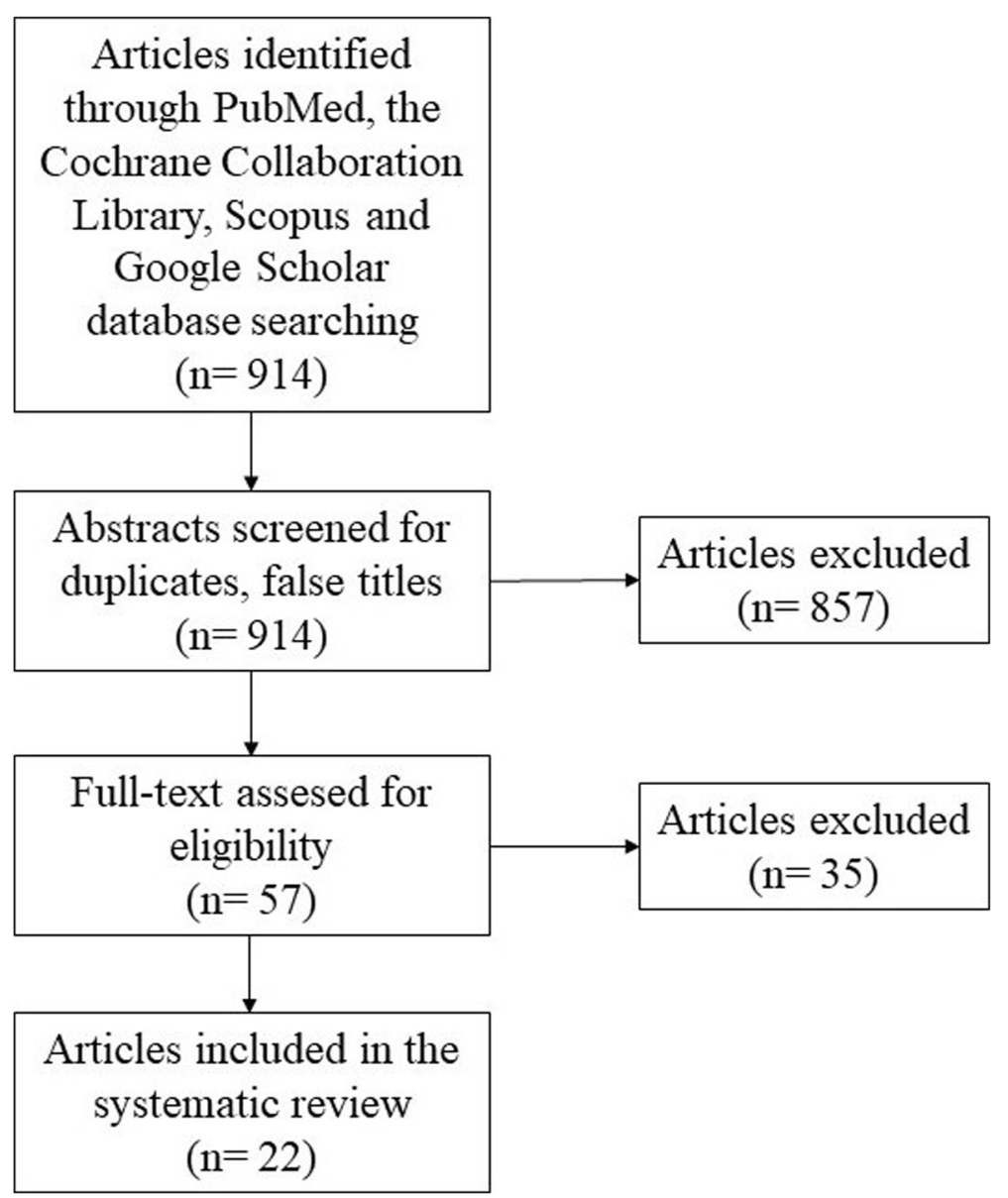

Fig. 1 PRISMA flow chart demonstrating the systematic review of the literature

27 months after surgery showed an improvement in motor function undergoing physiotherapy, but no change of the sensory deficits. HRNS revealed stable intraneural PN.

\section{Case 2}

A 16-year-old left-handed male patient presented with an intraneural PN of the left ulnar nerve. Symptoms lasted over 2 years, showing a progressive atrophy of the intrinsic muscles of the left hand without any sensory deficit. Electrophysiological testing revealed an impairment of the left ulnar nerve, HRNS and MRI of the left arm showed intraneural PN of the ulnar nerve with a length of about $15 \mathrm{~cm}$ reaching from the middle of the upper arm to the middle of the forearm (Fig. 3). Despite the atrophy of the intrinsic muscles of the hand, follow-up examination 24 months after surgery showed a stable state of the PN on HRNS, without any sensory deficit. The functionality of the hand improved under consequent physiotherapy.

\section{Case 3}

A 17-year-old female patient complained about progressive impairment of foot elevation over a period of 3 years, showing a paresis of $2 / 5$. The patient was treated by the neurologist due to the suspicious of an idiopathic peroneal paresis. Electrophysiological testing showed impairment of the left peroneal nerve. HRNS and MRI of the left leg revealed an intraneural PN of peroneal nerve (Fig. 4). Postoperative, the paresis of the foot elevation increased to $1 /$ 5. Follow-up examination 30 months after surgery showed an improvement of the foot elevation, now $3 / 5$.

\section{Histopathology}

Histology of the biopsy specimen showed intrafascicular tumour cells surrounded by collagen fibres. Epithelial membrane antigen staining highlights concentric layers of tumour cells. S100 is not expressed in tumour cells but positive in the preexistent Schwann cells. Immunohistochemical staining for Ki-67 reveals only very few proliferating tumour 

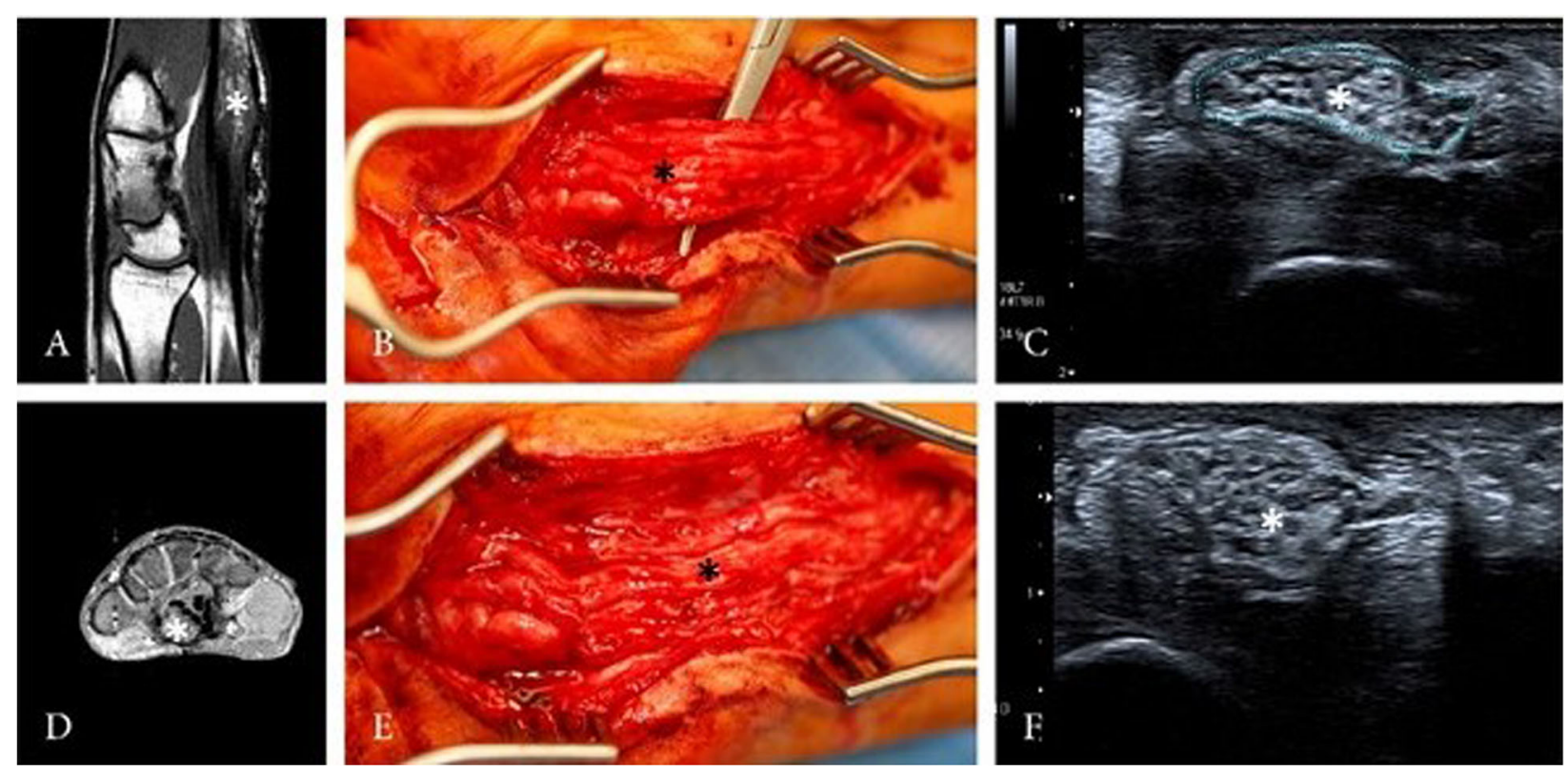

Fig. 2 HRNS $(\mathbf{c}+\mathbf{f})$ and MRI $(\mathbf{a}+\mathbf{d})$ of the right-hand depicted nerve enlargement (asterisk) with perineurial tissue and still identifiable fascicular structure. Intraoperative view showing the perineurioma $(\mathbf{b}+\mathbf{e})$

cells (Fig. 5). All cases showed a similar histomorphology and a comparable immunohistochemical expression profile, so that an intraneural PN was diagnosed in all cases.

\section{Results of the systematic review}

The search resulted in 914 articles, after analysing the title and abstract 857 articles were excluded. The full text of the remaining 57 articles was reviewed and 35 articles were then excluded. Therefore, 22 articles met the inclusion criteria $[5,9,12-31]$, comprising 74 cases of PN (Table 1).

Including our three cases, demographics, surgical treatment and the neurological outcome were analysed for 77 patients.
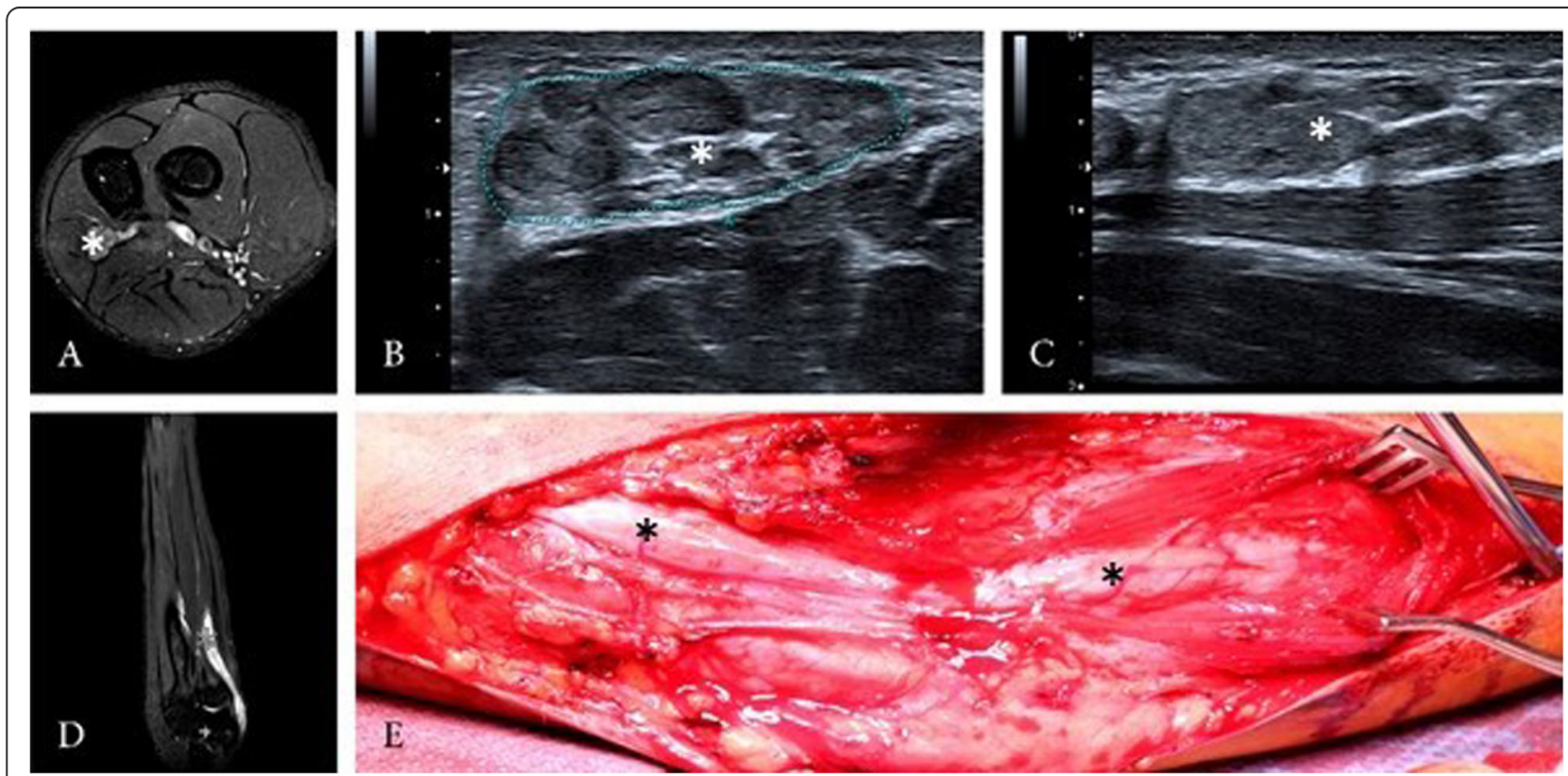

Fig. 3 HRNS $(\mathbf{b}+\mathbf{c})$ and MRI $(\mathbf{a}+\mathbf{d})$ of the left forearm depicted nerve enlargement (asterisk) with perineurial tissue and still identifiable fascicular structure. Intraoperative view showing the perineurioma (e) 

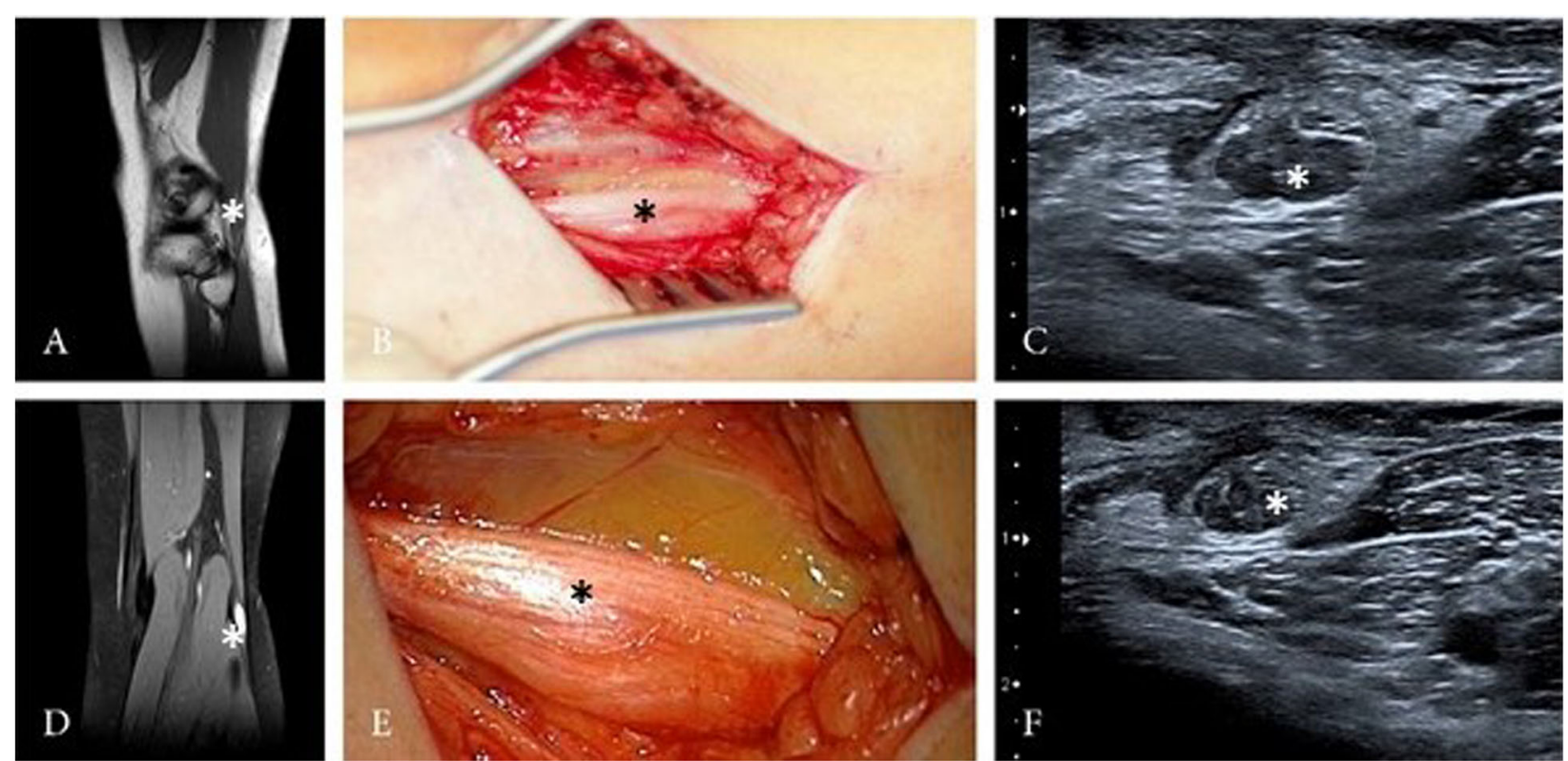

Fig. 4 HRNS $(\mathbf{c}+\mathbf{f})$ and MRI $(\mathbf{a}+\mathbf{d})$ of the left upper limb depicted nerve enlargement (asterisk) with perineurial tissue and still identifiable fascicular structure. Intraoperative view showing the perineurioma $(\mathbf{b}+\mathbf{e})$

\section{Demographics}

Mean age was $21.0 \pm 13.9$ years of age, 42 females (54.5\%). Mean follow-up was 35.2 months. Sciatic nerve was affected mostly $(n=16,20.8 \%)$, followed by peroneal $(n=11,14.3 \%)$ and median nerve affection $(\mathrm{n}=11,14.3 \%)$.

\section{High-resolution nerve sonography}

Typically, PN present as a fusiform enlargement of the nerve fascicles over several centimetres with hyperechoic perineurial tissue on HRNS. Fascicular structure of the enlarged nerves remains visible.

\section{Magnetic resonance imaging}

Normally, PN show isointensity on T1-weighted and hyperintensity on T2-weighted MRI. Intraneural PN can show highly increased signal along the nerve on ShortTau Inversion Recovery sequence with identifiable fascicles. Homogenous, moderate to strong contrast enhancement can be detected on T1-weighted contrast enhanced MRI.

\section{Surgery}

Biopsy was performed in 32 patients (41.6\%), complete resection and nerve grafting was performed in 28 cases (36.4\%), whereas neurolysis $(n=12,15.6 \%)$ or fascicular biopsy and interfascicular neurolysis $(n=5,6.5 \%)$ was performed less.

\section{Neurological outcome}

Neurological outcome was unchanged in 35 cases (45.5\%), worsened in 16 cases (20.8\%). Neurological improvement was detected in 26 patients (33.8\%).

Analysis of the review of the literature revealed similar results between the different surgical treatment options. Complete resection with nerve grafting showed unchanged neurological outcome in $46.4 \%$ and an improvement in $35.7 \%$. Single biopsy showed unchanged results of the neurological outcome in $53.1 \%$ and an improvement in $18.8 \%$. Unchanged neurological outcome was detected in $41.6 \%$ and improved neurological outcome was seen in $41.6 \%$ of the cases treated with neurolysis (Table 2).

\section{Discussion}

Intraneural PNs are rare benign peripheral nerve sheath tumours originating from perineurial cells, presenting slowly progressive, motor predominant focal neuropathy or plexopathy with mild sensory symptoms and signs [9].

Probably, intraneural $\mathrm{PN}$ is an under-diagnosed focal neuropathy, but more cases were detected over the last years due to a multidisciplinary approach by experts in peripheral nerve care, peripheral nerve imaging including HRNS, peripheral nerve surgery, electrophysiology and peripheral nerve pathology.

On MRI, PNs typically present as a fusiform enlargement of the nerve fascicles, isointense on T1-weighted and moderate to strong hyperintensity on T2-weighted MR images with moderate to strong homogenous contrast enhancement after intravenous gandolinium application $[9,32]$. On HRNS examinations, a nerve enlargement over 

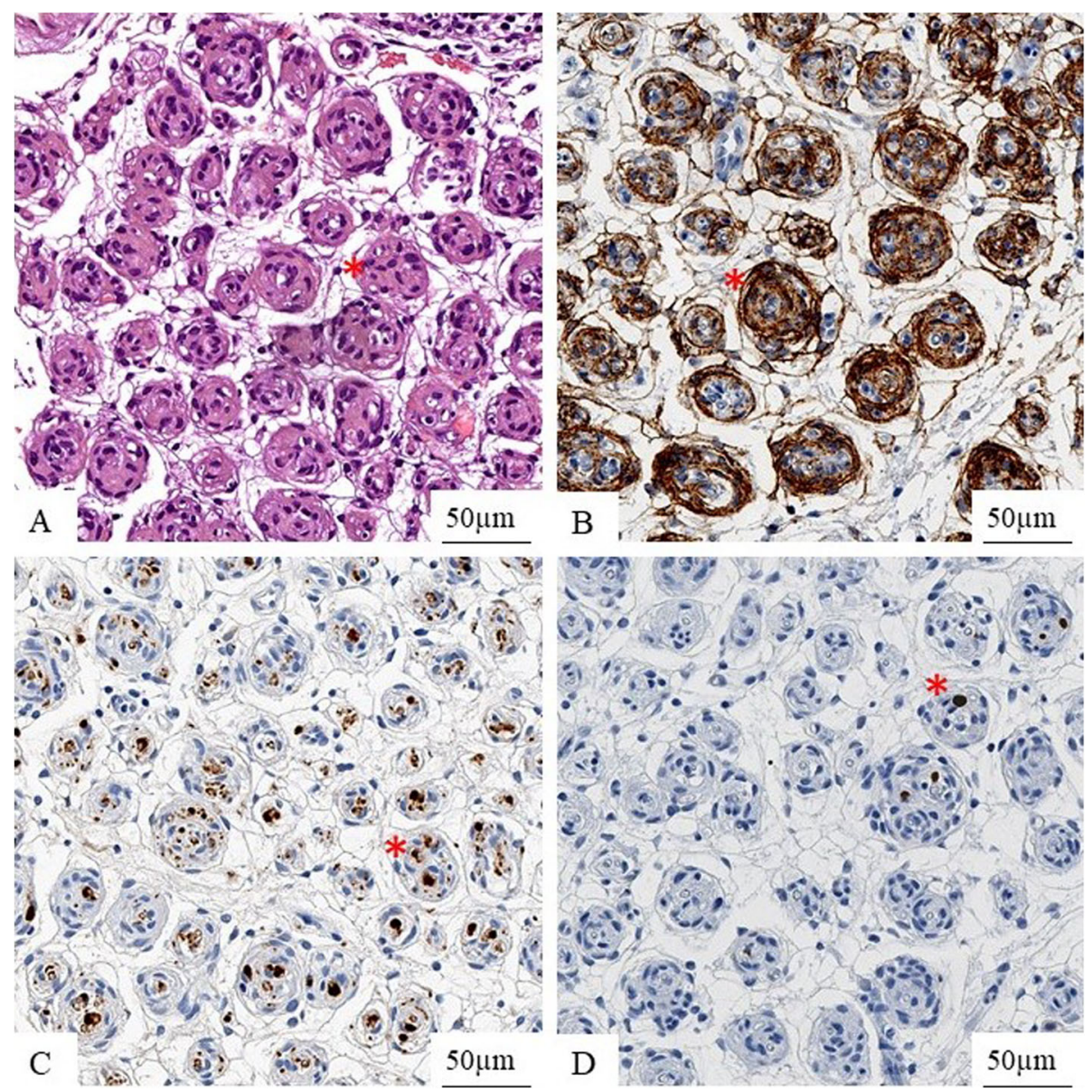

Fig. 5 Histology of the biopsy specimen (Hematoxylin and eosin stain) with intrafascicular tumour cells (asterisk) surrounded by collagen fibres (a). Epithelial membrane antigen staining is positive in tumour cells (asterisk) (b). S100 is not expressed in tumour cells but shows entrapped Schwann-cells in pseudo-onion bulbs (asterisk) (c). Immunohistochemical staining for Ki-67 reveals only very few proliferating tumour cells (asterisk) (d)

several centimetres with hyperechoic perineurial tissue can be detected. Fascicular structure of the enlarged nerves is still identifiable [10]. Up to date, HRNS is more and more commonly used for peripheral neuropathies [33], and is a well-established diagnostic tool, which can be used for follow-up examinations of known PNs. We believe that tumour growth can be detected easily with HRNS. Therefore, HRNS plays an important role in diagnosis and especially in follow-up examination in combination with the neurological examinations and the findings of the electrophysiological testing.

However, the treatment of intraneural PN is still a subject of great controversy despite several studies, mainly case reports, on this topic. A general consensus still does not exist. Surgery is commonly recommended in patients with a progressive neurological deficit and a localizable single lesion.

Gruen et al. recommended in their series of intraneural PNs that a surgical treatment should include resection of the lesion with interpositional nerve graft repair in cases where intraoperative nerve action potentials demonstrate a non-functioning or poorly functioning segment. Additionally, intraoperative histological examination is recommended to confirm onion- bulb neuropathy. They reported in their series, including 14 patients with localized hypertrophic neuropathy, the postoperative outcome after surgery. Seven out of $10 \mathrm{pa}-$ tients who received nerve grafting showed an improvement of the motor function. Four patients did not receive nerve grafting, but two of those patients recovered some function. Therefore, Gruen et al. concluded that routine nerve grafting should be performed [13].

Gruen and Kline also recommend that the lesion should be carefully resected until normal appearing fascicles and a bit further both proximally and distally of the lesion and nerve graft should be interposed, if no action potential or if poor amplitude was recorded across the lesion. More extensive nerve resection is made to avoid that the 


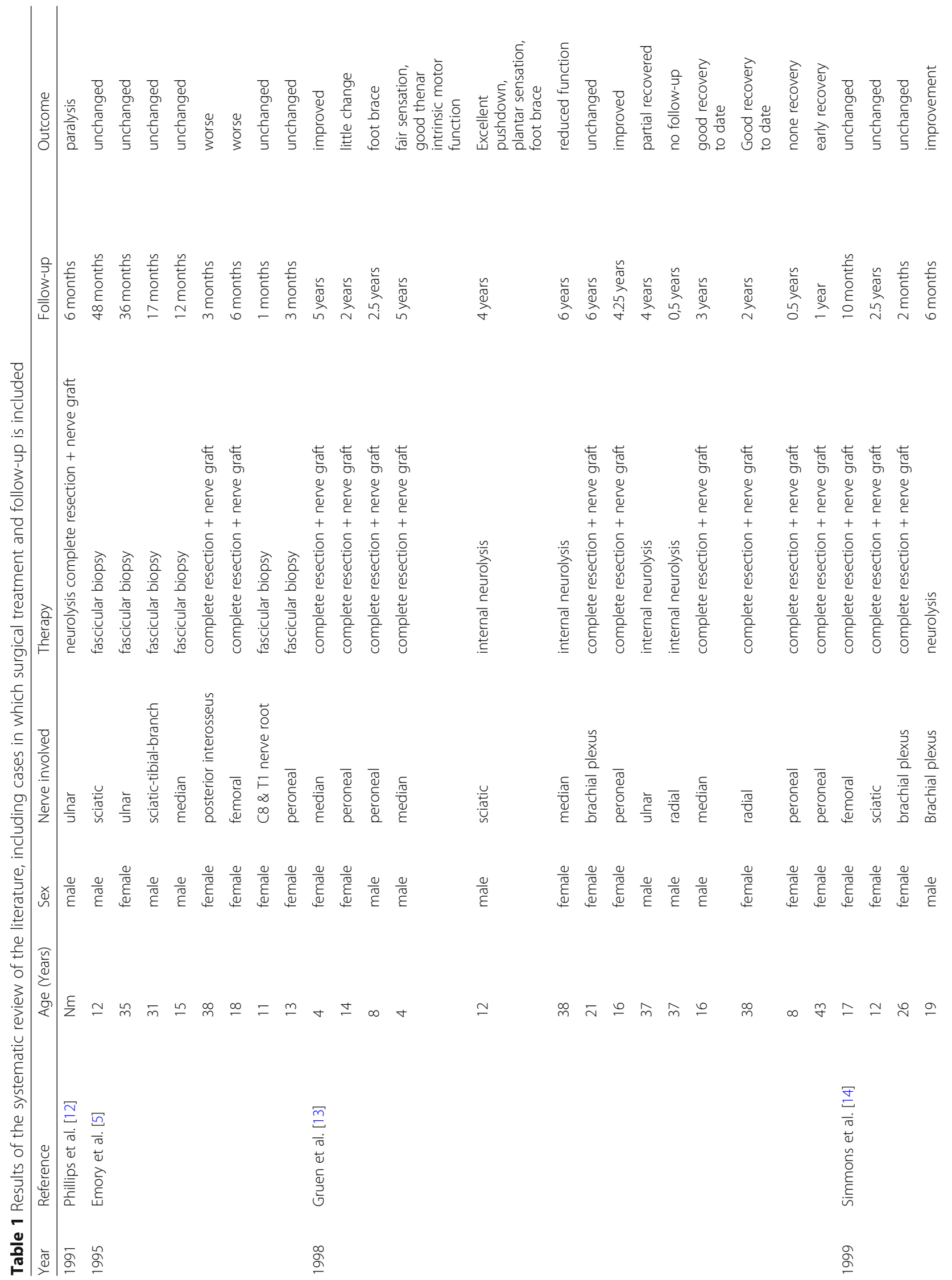




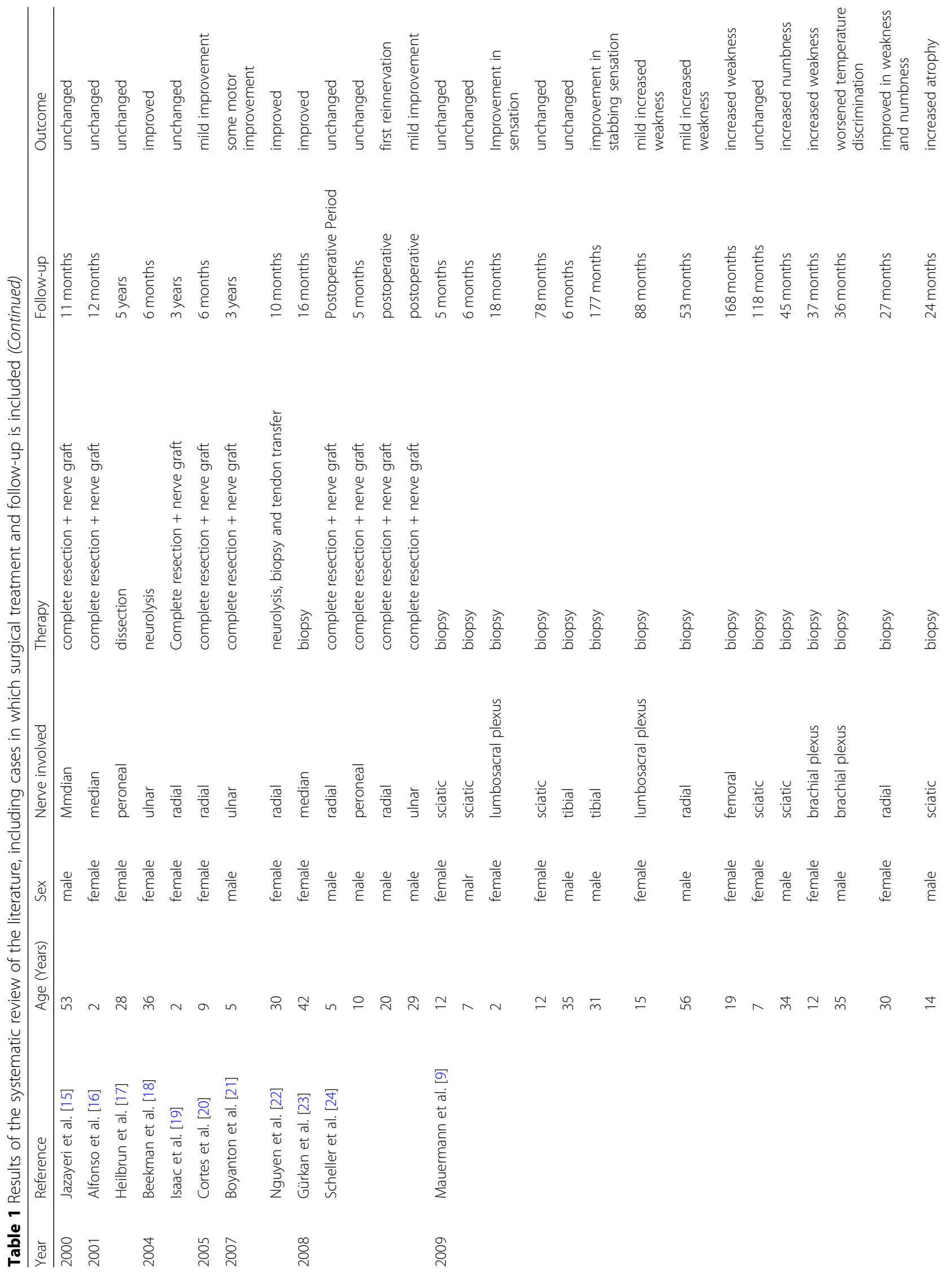




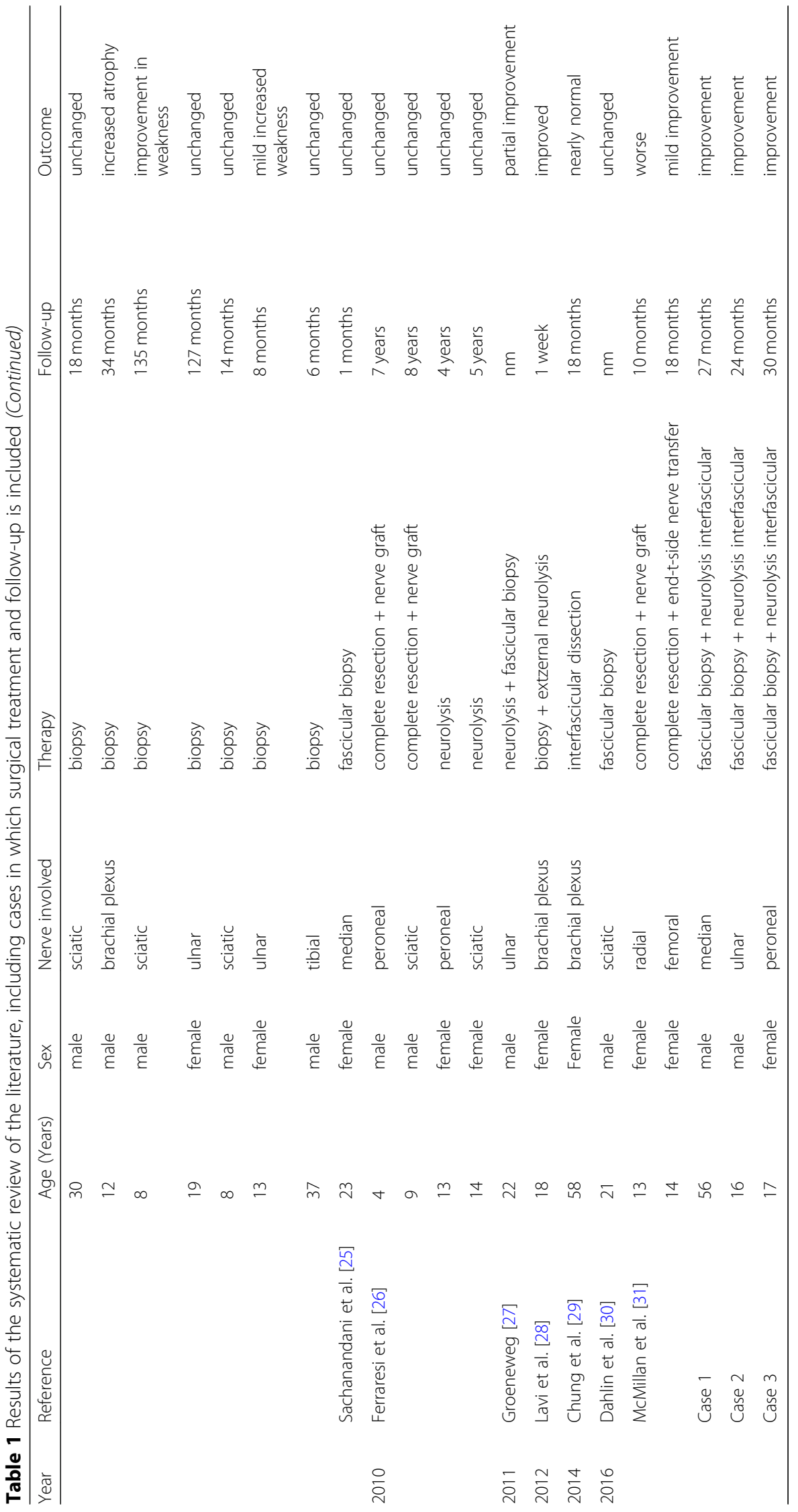


Table 2 Different surgical therapy and its neurological outcome

\begin{tabular}{llll}
\hline Surgical Treatment & \multicolumn{3}{l}{ Neurological outcome } \\
\cline { 2 - 4 } & Worsened & Unchanged & Improved \\
\hline Complete resection + nerve graft & $5(17.9 \%)$ & $13(46.4 \%)$ & $10(35.7 \%)$ \\
Biopsy & $9(28.1 \%)$ & $17(53.1 \%)$ & $6(18.8 \%)$ \\
Fascicular biopsy + neurolysis & 0 & 0 & $5(100 \%)$ \\
Neurolysis & $2(16.8 \%)$ & $5(41.6 \%)$ & $5(41.6 \%)$ \\
\hline
\end{tabular}

interpositioned nerve graft may eventually deteriorate as well, leading to a longer graft and therefore to poorer results [34]. In our opinion, extensive nerve resection and following nerve grafting has to be considered in patients with more distal located focal lesion without muscle atrophy. Otherwise, there is always the possibility of worsening disability or pain after surgical treatment of the nerve. Those criteria meet only in a few cases so that nerve resection should usually not be performed.

Heilbrun et al. reviewed the treatment options in patients with intraneural PN. They found 16 patients treated with resection of the tumour and nerve grafting. Collected follow-up information showed an improvement of motor function in eight cases and a slight improvement in two more patients. Three of five patients treated by tumour excision alone also demonstrated an improvement of the neurological deficits. They concluded that nerve grafting should be based on individually indications including negative intraoperative action potentials [17]. Early identification of the lesion and the treatment opportunity prior to irreversible deterioration of distal intramuscular nerve sheaths and longstanding denervation atrophy are also an important factor influencing the neurological outcome. Functional recovery after nerve grafting is most favourable in young patients [20].

Sachanandani et al. recommended as an alternative to proximal nerve grafting a distal nerve transfer in patients with progressive neuropathies due to several reasons. Widely resection of the lesion is possible using a nerve reconstruction that is distant to the lesion without any concern for the length of graft required [25]. Furthermore, reinnervation of the target musculature might be more successful to decreased length of regeneration required, elimination of an additional repair site, and exclusion of grafts [35]. Additionally, harvesting a nerve graft using a second surgery site and possible causing further deficits may be avoided [25].

Mauermann et al. present a dissenting opinion of the treatment of intraneural PN. In their opinion and current clinical practice, targeted fascicular nerve biopsy at the site of MRI lesion has to be performed as a much more focused nerve biopsy minimizing surgical deficits and postoperative scarring. Other surgical interventions such as tendon transfers or distal nerve grafting may be performed as a second stage procedure in patients there a static disease is evident after receiving the definitive diagnosis. Furthermore, clinically follow-up with imaging to verify clinical stability may be reasonable in patients there the nerve lesion is difficult to access without major invasive surgery [9].

We are in line with the therapeutically approach performing a targeted fascicular nerve biopsy at the site of MRI or HRNS lesion, but in our cases additionally interfascicular neurolysis, which is known to be a safe and reliable technique [36] had a positive impact on the neurological outcome.

Contrary to the common view and contrary to our approach, Restrepo et al. believe that a targeted fascicular biopsy is not necessary in patients there the clinical presentation (slowly progressive painless neuropathy and motor dysfunction with atrophy in the affected nerve territory) and the radiological imaging are typical for the presence of intraneural PN [37]. In our opinion, a targeted biopsy under electrophysiological monitoring is mandatory to receive a definitive diagnosis even in cases there the clinical presentation and the radiological imaging is highly suspicious of an intraneural PN to avoid unnecessary treatment.

However, neurological outcome did not differ between the analysed surgical treatment options (Table 2). Radiological evaluations of PN revealed more information about the course of this rare tumour. This might shift the surgical treatment from total resection and nerve grafting towards less invasive methods like targeted fascicular biopsy for diagnostic and wait-and-see strategies. This was suggested by Wilson et al., who were able to show that intraneural PN rarely growth in length and that they do not grow to involve new nerves or nerve divisions. Furthermore, growth does not correlate with clinical progression. Therefore, they concluded that intraneural PN have special characteristics, which can be detected radiological and which would make invasive diagnostic unnecessary [38].

Several limitations have to be addressed. First, this is a systematic review about a rare tumour. Therefore, we were only able to review case reports and small case studies. The informative value of those low evidence articles must be kept in mind. Secondly, neurological outcome was described only for several months.

However, future prospective studies with a longer follow-up are needed to evaluate the neurological longterm outcome in those patients.

\section{Conclusion}

Intraneural $\mathrm{PN}$ is rare benign slowly progressive nerve sheath tumour. Treatment management is still controversy discussed but seems to become more and more less invasive. At least, targeted biopsy should be performed to receive histopathological diagnosis. HRNS is increasingly used for follow-up examinations. 


\section{Abbreviations}

HRNS: High resolution nerve sonography; MRI: Magnetic resonance imaging; PN: Perineurioma

\section{Acknowledgements}

We acknowledge support by the Open Access Publication Fund of the University of Duisburg-Essen.

\section{Authors' contributions}

All authors contributed to the study conception and design. Material preparation, data collection and analysis were performed by OG and A-KU. The first draft of the manuscript was written by OG, A-KU and CK. All authors commented on previous versions of the manuscript. Review and editing were performed by KHW and US. AJ provided the histological pictures. All authors read and approved the final manuscript.

\section{Funding}

An IFORES grant (D/107-40960) to Oliver Gembruch from the University of Duisburg-Essen supported the research. The sponsor had no role in the design or conduct of this research.

\section{Availability of data and materials}

All data generated or analysed during this study are included in this published article.

\section{Ethics approval and consent to participate}

The study has been carried out in accordance with The Code of Ethics of the World Medical Association (Declaration of Helsinki) and was approved by the Institutional Review Board (Medical Faculty, University of Duisburg-Essen, Registration number: 18-7955-BO). Consent to paticipate was obtained from each patient.

\section{Consent for publication}

Written informed consent was obtained from each patient for publication of this article.

\section{Competing interests}

The authors declare that they have no competing interests.

\section{Author details}

${ }^{1}$ Department of Neurosurgery, University Hospital Essen, University of Duisburg-Essen, Essen, Germany. ${ }^{2}$ Department of Neurology, St. Josef-Hospital Bochum, Ruhr University Bochum, Bochum, Germany. ${ }^{3}$ Department of Neuropathology, University Hospital Essen, University of Duisburg-Essen, Essen, Germany.

\section{Received: 13 August 2019 Accepted: 6 February 2020}

\section{Published online: 13 February 2020}

\section{References}

1. Macarenco RS, Ellinger F, Oliveira AM. Perineurioma: a distinctive and underrecognized peripheral nerve sheath neoplasm. Arch Pathol Lab Med. 2007:131(4):625-36

2. Ariza A, Bilbao J, Rosai J. Immunohistochemical detection of epithelial membrane antigen in normal perineurial cells and perineurinoma. Am J Surg Pathol. 1988;12:678-83.

3. Rodriguez FJ, Folpe AL, Giannini C, Perry A. Pathology of peripheral nerve sheath tumors: diagnostic overview and update on selected diagnostic problems. Acta Neuropathol. 2012;123(3):295-319.

4. Shanthaveerappa TR, Bourne GH. Perineural epithelium: a new concept of its role in the integrity of the peripheral nervous system. Science. 1966; 154(3755):1464-7.

5. Emory TS, Scheithauer BW, Hirose T, Wood M, Onofrio BM, Jenkins RB. Intraneural perineurioma. A clonal neoplasm associated with abnormalities of chromosome 22. Am J Clin Pathol. 1995;103(6):696-704.

6. Giannini C, Scheithauer BW, Jenkins RB, Erlandson RA, Perry A, Borell TJ, et al. Soft-tissue perineurioma. Evidence for an abnormality of chromosome 22, criteria for diagnosis, and review of the literature. Am J Surg Pathol. 1997;21(2):164-73.
7. Klein CJ, Wu Y, Jentoft ME, Mer G, Spinner RJ, Dyck PJ, et al. Genomic analysis reveals frequent TRAF7 mutations in intraneural perineuriomas. Ann Neurol. 2017:81(2):316-21.

8. Carter JM, Wu Y, Blessing MM, Folpe AL, Thorland EC, Spinner RJ, et al. Recurrent genomic alterations in soft tissue Perineuriomas. Am J Surg Pathol. 2018:42(12):1708-14.

9. Mauermann MLAK, Kuntz NL, Spinner RJ, Dyck PJ, Bosch EP, Engelstad J, Felmlee JP, Dyck PJ. Longitudinal study of intraneural perineurioma--a benign, focal hypertrophic neuropathy of youth. Brain. 2009;132:2265-76.

10. Krogias C, Gold R, Schelle T, Bohm J, Junker A, Sure U, et al. Teaching Neurolmages: Sonographic detection of intraneural perineurioma in therapy-refractory carpal tunnel syndrome. Neurology. 2017;88(10):e85-e6.

11. Moher D, Liberati A, Tetzlaff J, Altman DG, Group P. Preferred reporting items for systematic reviews and meta-analyses: the PRISMA statement. Ann Intern Med. 2009:151(4):264-9 W64.

12. Phillips LH 2nd, Persing JA, Vandenberg SR. Electrophysiological findings in localized hypertrophic mononeuropathy. Muscle Nerve. 1991;14(4):335-41.

13. Gruen JP, Mitchell W, Kline DG. Resection and graft repair for localized hypertrophic neuropathy. Neurosurgery. 1998;43(1):78-83.

14. Simmons Z, Mahadeen Zl, Kothari MJ, Powers S, Wise S, Towfighi J. Localized hypertrophic neuropathy: magnetic resonance imaging findings and long-term follow-up. Muscle Nerve. 1999;22(1):28-36.

15. Jazayeri MA, Robinson JH, Legolvan DP. Intraneural perineurioma involving the median nerve. Plast Reconstr Surg. 2000;105(6):2089-91.

16. Alfonso DT, Sotrel A, Grossman JA. Carpal tunnel syndrome due to an intraneural perineurioma in a 2-year-old child. J Hand Surg Br. 2001;26(2):168-70.

17. Heilbrun ME, Tsuruda JS, Townsend JJ, Heilbrun MP. Intraneural perineurioma of the common peroneal nerve. Case report and review of the literature. J Neurosurg. 2001;94(5):811-5

18. Beekman R, Slooff WB, Van Oosterhout MF, Lammens M, Van Den Berg LH. Bilateral intraneural perineurioma presenting as ulnar neuropathy at the elbow. Muscle Nerve. 2004:30(2):239-43.

19. Isaac S, Athanasou NA, Pike M, Burge PD. Radial nerve palsy owing to localized hypertrophic neuropathy (intraneural perineurioma) in early childhood. J Child Neurol. 2004;19(1):71-5.

20. Cortes W, Cheng J, Matloub HS. Intraneural perineurioma of the radial nerve in a child. J Hand Surg Am. 2005;30(4):820-5.

21. Boyanton BL Jr, Jones JK, Shenaq SM, Hicks MJ, Bhattacharjee MB. Intraneural perineurioma: a systematic review with illustrative cases. Arch Pathol Lab Med. 2007;131(9):1382-92.

22. Nguyen D, Dyck PJ, Daube JR. Intraneural perineurioma of the radial nerve visualized by 3.0 tesla MRI. Muscle Nerve. 2007;36(5):715-20.

23. Gurkan V, Sever G, Karaca G, Orhun H, Bilgic B. Re: intraneural perineurioma of the median nerve. A rare cause of carpal tunnel syndrome. J Hand Surg Eur Vol. 2008;33(1):88-9.

24. Scheller C, Richter HP, Scheuerle A, Kretschmer T, Konig RW, Antoniadis G. Intraneural perineuriomas; a rare entity. Clinical, surgical and neuropathological details in the management of these lesions. Zentralb| Neurochir. 2008:69(3):134-8.

25. Sachanandani NS, Brown JM, Zaidman C, Brown SS, Mackinnon SE. Intraneural perineurioma of the median nerve: case report and literature review. Hand (N Y). 2010;5(3):286-93.

26. Ferraresi S, Garozzo D, Bianchini E, Gasparotti R. Perineurioma of the sciatic nerve: a possible cause of idiopathic foot drop in children: report of 4 cases. J Neurosurg Pediatr. 2010:6(5):506-10.

27. Groeneweg AJ, Hartman EH, Fleischeuer R, Visser LH. An unusual location of ulnar nerve pathology: a perineurioma of the ulnar nerve in the upper arm. Muscle Nerve. 2011;44(4):593-6.

28. Lavi ES, Levi AD, Schallert EK, Brown AD, Norenberg MD. MRI of intraneural perineurioma of the brachial plexus. Radiol Case Rep. 2012; 7(1):649.

29. Chung JH, Jeong SH, Dhong ES, Han SK. Surgical removal of intraneural perineurioma arising in the brachial plexus using an interfascicular dissection technique. Arch Plast Surg. 2014:41(3):296-9.

30. Dahlin LB, Nennesmo I, Besjakov J, Ferencz I, Andersson GS, Backman C. Case report: Intraneural perineurioma of the sciatic nerve in an adolescent - strategies for revealing the diagnosis. Clin Case Rep. 2016; 4(8):777-81.

31. McMillan HJ, Torres C, Michaud J, Ying Y, Boyd KU, Bourque PR. Diagnosis and outcome of childhood perineurioma. Childs Nerv Syst. 2016;32(8):1555-60. 
32. Wilson TJ, Howe BM, Stewart SA, Spinner RJ, Amrami KK. Clinicoradiological features of intraneural perineuriomas obviate the need for tissue diagnosis. J Neurosurg. 2018;129(4):1034-40.

33. Kerasnoudis A, Tsivgoulis G. Nerve ultrasound in peripheral neuropathies: a review. J Neuroimaging. 2015;25(4):528-38.

34. Gruen P, Kline DG. Hypertrophic mononeuropathy. Neurosurg Focus. 2007;22(6):E23.

35. Brown JM, Shah MN, Mackinnon SE. Distal nerve transfers: a biology-based rationale. Neurosurg Focus. 2009;26(2):E12.

36. Mackinnon SE, O'Brien JP, Dellon AL, McLean AR, Hudson AR, Hunter DA. An assessment of the effects of internal neurolysis on a chronically compressed rat sciatic nerve. Plast Reconstr Surg. 1988;81(2):251-8.

37. Restrepo CE, Amrami KK, Howe BM, Dyck PJ, Mauermann ML, Spinner RJ. The almost-invisible perineurioma. Neurosurg Focus. 2015;39(3):E13.

38. Wilson TJ, Amrami KK, Howe BM, Spinner RJ. Clinical and radiological follow-up of Intraneural Perineuriomas. Neurosurgery. 2019;85(6):786-92.

\section{Publisher's Note}

Springer Nature remains neutral with regard to jurisdictional claims in published maps and institutional affiliations.

Ready to submit your research? Choose BMC and benefit from:

- fast, convenient online submission

- thorough peer review by experienced researchers in your field

- rapid publication on acceptance

- support for research data, including large and complex data types

- gold Open Access which fosters wider collaboration and increased citations

- maximum visibility for your research: over $100 \mathrm{M}$ website views per year

At $\mathrm{BMC}$, research is always in progress.

Learn more biomedcentral.com/submissions 